\title{
Estimation of Unmodeled Gravitational Wave Transients with Spline Regression and Particle Swarm Optimization
}

\author{
Calvin Leung \\ Harvey Mudd College, 340 E. Foothill Blvd., Claremont, CA 91711; cleung@hmc. edu* \\ Project supervisor: Soumya D. Mohanty \\ Dept. of Physics and Astronomy, University of Texas at Brownsville, \\ One W. University Blvd., Brownsville, TX 78520; soumya. mohanty@utb. edu
}

\begin{abstract}
Detecting and estimating unmodeled transient gravitational wave (GW) signals in noisy data is a major challenge in GW data analysis. This paper explores a solution that combines spline based regression with Particle Swarm Optimization for knot placement and directional parameter estimation. First, the estimation of binary black hole merger signals in data from a single GW detector is used as a testbed problem to quantify the relative performance of several algorithmic design choices. The method resulting from this study is then adapted to the case of data from a network of geographically distributed GW detectors. Simulation results show fairly good directional estimates for black hole mergers, with reasonable fidelity in the reconstruction of both GW polarization waveforms, at a signal to noise ratio capped at 15 for any single detector in the network. This promising performance suggests that the method should be developed further and applied to other types of GW transients.
\end{abstract}

\section{INTRODUCTION}

Gravitational waves are a fundamental prediction of Einstein's general theory of relativity [29]. They are perturbations in the metric of space-time that propagate with the speed of light and are sourced by time dependent changes in mass-energy distribution. GWs have exceedingly weak interaction with matter, making them difficult to detect. However, being able to meet this challenge will open up a new way of observing the Universe. This is expected to revolutionise our understanding of many hitherto hidden astrophysical phenomena such as the dynamics of core-collapse supernovae [20].

A decades-long world-wide effort has been going on to build instruments capable of directly detecting GWs incident on Earth from astrophysical sources. (Such an instrument needs to be able to measure length changes of $\sim 10^{-18} \mathrm{~m}$ over $\sim 1 \mathrm{~km}$ !) This effort crossed a major milestone recently with the successful demonstration of the LIGO detectors [1] running stably at their planned initial sensitivity in coincidence with the FrenchItalian Virgo [2] and the German-British GEO600 [16] detectors. The LIGO detectors are currently undergoing upgrades[18], with a nominal date of completion in 2015, that will boost the distance to which an astrophysical source can be seen by a factor of $\sim 10$. The Virgo detector is undergoing similar upgrades [9] and a comparable sensitivity cryogenic detector called KAGRA [3] is under construction in Japan. Based on astrophysical rate estimates of sources, it is believed that these advanced detectors will be sensitive enough to detect several GW signals of astrophysical origin per year. By combining data from a world wide network of detectors, we will be

\footnotetext{
* Initially published in the LIGO DCC as LIGO-T1400565.
}

able to estimate the location of a GW source on the sky and its physical parameters [21].

Even with the impressive sensitivity of advanced GW detectors, a typical GW signal will not stand out from the instrumental noise background. Sophisticated signal processing methods must be employed to search for GW signals in noisy data and estimate their properties. A particularly hard challenge in GW data analysis is posed by unmodeled signals whose waveforms are unknown to us a priori - for example, GWs produced by turbulent dynamics in core-collapse supernovae [25]. Another example is the merger of two black holes where the GW signal can in principle be calculated from numerical relativity simulations but where the computational cost makes a complete coverage of the parameter space of such signals infeasible [26].

Maximum likelihood estimation under a smoothness regularization condition $[13,17]$ is a promising new approach $[23]$ to the detection and estimation of unmodeled transient GW signals. This scheme naturally leads to the regression spline method [14] in which the estimated signals are cubic spline functions. Finding the best fit signal in this method is a challenging non-linear and highdimensional optimization problem, but this problem can be successfully addressed [15, 23] using Particle Swarm Optimization (PSO) [11]. First applications [19] of the PSO based regression spline method to the estimation of black hole merger signals in a single detector showed promising results. A straightforward extension of this method was made to the case of a network of GW detectors [22].

This paper explores several design choices possible in the PSO based regression spline approach. We use a set of performance metrics and extensive simulations, on the binary black hole merger testbed, to study the impact of these design choices. A generalization of the resulting method to coherent network analysis is proposed and pre- 
liminary results are obtained regarding the accuracy of directional estimation and reconstruction of the GW polarization waveforms. We find that the method developed in this paper performs well and that the time-domain spline-based approach should be developed further and applied to a wider variety of GW signals.

The rest of the paper is organized as follows. Sec II provides a brief review of technical background behind the PSO based regression spline method. Sec. III describes the simulation pipeline as well as the metrics used in this paper for quantifying the performance of the method. The various design choices possible in this approach are presented in Sec. IV along with a quantitative study of their impact on performance. Sec. V presents the generalization of the final method to the case of network analysis and preliminary results on its performance. Our conclusions are contained in Sec. VI.

\section{BACKGROUND}

A spline is a piecewise polynomial function obeying some smoothness conditions at the junctions (called knots) where the pieces connect. For a given set of knots, all splines of a given degree of the polynomial pieces constitute a linear vector space. A convenient basis for this space is that of B-splines [8], which are themselves linearly independent, piecewise polynomials. In the following, we will restrict ourselves to the case of cubic splines defined by a fixed number, $k$, of knots confined to a finite time interval $\left[T_{0}<t_{1}, t_{k}<T_{f}\right.$ ], where $T_{0}$ and $T_{f}$ denote the start and end time of a time series data segment from a single detector.

The set of cubic B-splines is unique for each partition of the domain $\left(t_{1} \leq t_{2} \leq \ldots t_{k-1} \leq t_{k}\right)$. Furthermore, if the partition is strictly increasing such that $\left(t_{1}<t_{2}<\ldots t_{k-1}<t_{k}\right)$, then the partition's corresponding B-splines, and therefore any spline, will by construction be $\mathscr{C}^{2}$ continuous. In the case that the partition is uniformly spaced, i.e. $t_{i}-t_{i-1}=t_{i+1}-t_{i}$ for $i=2, \ldots k-1$, the interior B-splines are shifted copies of each other, reducing computational time in implementations of a spline-fitting algorithm.

Splines offer a flexible model to fit a wide variety of GW signals in noisy, oscillatory data; furthermore the fact that the set of all splines given a domain partition are linear combinations of the corresponding B-splines makes least-squares fitting straightforward: once the partition $P=\left(t_{1}<t_{2}<\ldots t_{k-1}<t_{k}\right)$ is fixed, it is well known that the least-squares spline best fitting the data $D(t)$ can be analytically found by minimizing (1).

$$
R(\vec{\alpha})=\sum_{i=1}^{N}\left(D\left(t_{i}\right)-\sum_{j=1}^{k-3} \alpha B_{j}\left(t_{i}\right)\right)^{2}
$$

where $\alpha_{j}$ denotes the scalar weightings of the B-Spline $B_{j}$.
In general, however, the effectiveness of splines in fitting transient signals is sensitive to the placement of the knots. For example, the location of the signal in time is unknown, so we need to find the partition $P$ such that the supported domain of the spline $\left[t_{1}, t_{k}\right]$ roughly coincides with the transient signal. The search for a viable partition is a challenging high-dimensional, nonlinear, and multimodal optimization problem and the effectiveness of the spline based fitting approach is critically dependent on solving this problem successfully. This is where the PSO algorithm can play a crucial role for its robustness in the face of these three challenges, relative simplicity, and ease of tuning compared to other nonlinear optimization/evolutionary algorithms.

PSO aims to minimize a scalar fitness function $f(\vec{x})$ defined over some search space $X$. First, many candidate solution vectors, or particles, $\vec{x}_{i} \in X, i=1,2, \ldots, N_{p}$, are randomly initialized with some random "velocities" $\vec{v}_{i}$. The particles 'swarm' stochastically throughout the search space searching for its minimum until some stop condition is met. The dynamical equation is simple: the $i$-th particle's velocity at the $j+1$-th time step is

$$
\vec{v}_{i}[j+1]=m \vec{v}_{i}[j]+c_{1} R_{1}\left(\vec{p}_{i}-\vec{x}_{i}[j]\right)+c_{2} R_{2}\left(\vec{q}_{i}-\vec{x}_{i}[j]\right)
$$

The three contributions to the particle's future velocity are as follows: the term $m \vec{v}_{i}[j]$ is the particle's current velocity scaled by $m$, which can be thought of the particle's "inertia". The second term pulls the particle towards $\vec{p}_{i}$, which is the minimizer of $f$ over all locations visited by the $i^{t h}$ particle. This can be thought of as the particle's "memory". The last term, considered the "social" velocity contribution, pulls the particle toward $\vec{q}_{i}$, which is the minimizer of $f$ over all locations visited by some subset of all the particles $N_{i} \subseteq P$. In general, the subset $N_{i}$ varies from particle to particle, and is called a neighborhood in the PSO literature. The $R_{i}$, which are random diagonal matrices with elements drawn from $U(0,1)$, randomly scale each component of the "memory" and the "social" contributions to add stochasticity to the algorithm. The parameters $m, c_{1}, c_{2}$ are scalars which are tuned to balance between a more-complete exploration of the full search space and the algorithm's convergence probability/rate. In the simplest PSO algorithms, the equation of motion is iterated over a fixed number of time steps $T_{s}$ specified by the user. At the end, the best of the best solutions found, namely the minimizer of $f\left(\vec{q}_{i}\right)$, $i=1,2, \ldots, N_{p}$, is returned.

Several variations of the basic PSO algorithm outlined above are possible [11]; furthermore there are general guidelines but none strictly dominates over all others in performance over all optimization problems[30]. We stay close to the version favored by Bratton and Kennedy [6]. For the parameters of the PSO algorithm in this paper, we use $c_{1}=c_{2}=2.05$ and an $m$ which linearly decreases from 0.9 to a final inertia 0.4 at the last time step. This standard parameter prescription [6] guarantees convergence while striking a good balance between exploration and convergence time. 
The choice of topology, i.e., how the neighborhoods $N_{j}$ are defined, plays an important role in the application of PSO to the regression spline method. We expand on this more later in the paper.

To apply PSO to find a suitable partition for spline regression, a transformation must first be defined that maps a domain partition to a vector $\vec{x}$ in a search space, typically a unit hypercube in $k$ dimensions, which contains all possible domain partitions. Then, a scalar fitness function is defined over the search space such that the spline corresponding to the best partition minimizes the fitness function. For example, our fitness function is one of least squares:

$$
R(\vec{x})=\sum_{i=1}^{N}\left(D\left(t_{i}\right)-s_{\vec{x}}\left(t_{i}\right)\right)^{2}
$$

Evaluation of the PSO fitness function 3 involves first transforming the search space location $\vec{x}$ to a partition $P$, analytically generating some best fit spline $s_{\vec{x}}(t)$, and computing the integrated square error.

\section{SIMULATION PIPELINE AND METRICS}

To characterize and refine the PSO based regression spline method, metrics for performance were defined and a simulation pipeline was set up in MATLAB. A representative binary black-hole merger signal was used, taken from AstroGravS [4], a database hosting GW waveforms obtained from numerical relativity simulations. Then, a varying amount of silence was added before and after the signal to simulate the unknown location of the signal in time. White Gaussian noise was added with a variance such that the signal to noise ratio (SNR), defined as

$$
S N R=\frac{\left(\int_{T_{0}}^{T_{f}} s(t)^{2} d t\right)^{1 / 2}}{R M S(n(t))}
$$

was between 7 and 10 depending on the test being run. Here, $s(t)$ and $n(t)$ are the signal and noise components of the data respectively. A PSO algorithm is then used to optimize over the set of all possible partitions of the time domain in the dataset, with the objective of finding the signal and producing an accurate, physically reasonable least-squares spline. This result is then compared to the pure signal using the following metrics.

Ideally, the PSO algorithm would (1) produce a partition which is close to the location of the true wave in the time domain, (2) return a spline which is a close approximation of the true wave and (3) converge to the same minimum reliably within a margin of error over multiple runs.

Three straightforward metrics shed light on the fulfillment of these objectives respectively. An ideal search for a suitable partition would minimize the following metric:

$$
K=\left(\frac{k-k^{\prime}}{k}\right)^{2}+\left(\frac{r^{*}-r}{r^{*}}\right)^{2}
$$

Let $k^{\prime}$ be the number of knots in the subinterval $\left[T_{0}^{*}, T_{f}^{*}\right]$ which corresponds to the true location of the GW transient and is a subinterval of the data defined over $\left[T_{0}, T_{f}\right]$. The first squared term is the square of the fraction of knots $t_{1}, t_{2}, \ldots t_{k}$ which fall in this correct interval. In the second term, $r^{*}$ denotes the true duration of the transient, while $r$ denotes the estimated duration of the transient, which is simply $t_{k}-t_{1}$.

The second metric compares the final best-fit spline to the pure signal used to generate the data via leastsquares:

$$
J[s]=\sum_{i=1}^{N}\left(s\left(t_{i}\right)-s_{0}\left(t_{i}\right)\right)^{2}
$$

where $s(t)$ is the spline estimate of the true signal $s_{0}(t)$.

The third metric is the fitness output of the PSO itself and is used comparatively between PSO runs on the same data. The location of the global minimum value of the fitness function is unknown in general, but the value itself is independent of the PSO design parameters and only depends on the dataset and the fitness function. Therefore, if multiple independent PSO runs on the same dataset converge to very similar fitness values, it is increasingly likely that the location found by PSO is close to the global minimum of the function.

\section{ALGORITHM DESIGN STUDY}

Possible improvements to the data pipeline were characterized. Four datasets, each with a unique time of arrival, were generated. The same representative merger signal at SNR $=7$ defined over $N$ data points. Each data stream lasted for a constant duration $T_{f}-T_{0}=$ $3\left(T_{f} *-T_{0} *\right)$. The four different times of arrival were $T_{0}^{*}=T_{0}, T_{0}+2 / 3\left(T_{0}-T_{f}\right), T_{0}+4 / 3\left(T_{0}-T_{f}\right), T_{0}+6 / 3\left(T_{0}-\right.$ $\left.T_{f}\right)$. The metrics for search accuracy, estimation accuracy, and convergence consistency were obtained via a full factorial study over three potential binary parameters, summarized in the following table and explained below. Paired t-tests on the three metrics were performed to reveal significant differences in PSO performance, with a total of $2^{3}$ possible parameter sets $\times 2$ possible termination times $\times 4$ times of signal arrival with a sample size of 64 trials.

\section{A. Knot Spacing}

A partition defined by $k$ knots is in general represented by a $k$ dimensional vector in a $k$ dimensional search space which must be searched by PSO. If the SNR is high 


\begin{tabular}{|l|r|r|}
\hline Parameter & Control & Experimental \\
\hline Spacing & Unconstrained & Linear \\
\hline Regularization & $\lambda=0$ & arg $\min _{\lambda>0}\{G C V(\lambda)\}$ \\
\hline Topology & gbest & Ring lbest \\
\hline$T_{\text {stop }}$ & N $/ \mathrm{A}$ & 50,250 time steps \\
\hline
\end{tabular}

TABLE I. The $T_{\text {stop }}$ values were chosen in an ad-hoc method by looking at the settling of the best fitness value to a minimum over time.
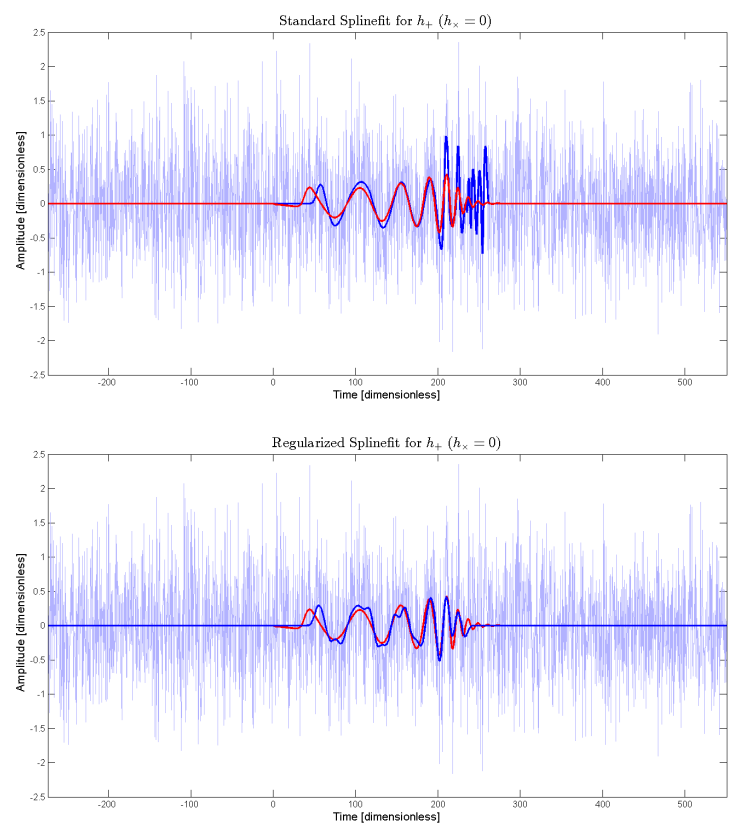

FIG. 1. The true signal injection (red) is contrasted with the spline (blue), which was estimated from the same set of noisy data (gray) containing the transient signal at $\mathrm{SNR}=$ 7. An unconstrained knot spacing with standard least-squares fitting is used on top, while a constrained knot spacing is used below with a regulated spline fit.

enough, the additional flexibility of the additional dimensions can improve accuracy of the fitting algorithm. However, the danger lies in lower SNRs, where this additional flexibility leads to overfitting of the data and clustering of knots, which results in flagrantly unphysical spline estimates and reduces the $\mathscr{C}^{2}$ smoothness of the spline. Fixing the number of knots and constraining the spacing between knots to be equal is one possible way to address overfitting. Since there are only two knot parameters to optimize (the location of the first and last knots), the dimensionality of the search space is reduced to 2 . Furthermore, calculations involving cubic B-splines can be simplified since closed-form expressions for B-splines exist in the case where the partition is equally-spaced and because the interior B-splines will all be shifted copies of each other.

Since there are only two free parameters that need to be numerically optimized in the constrained knots case, namely the two end knots, it is possible to explore the

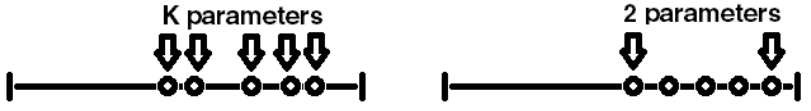

FIG. 2. The parameters to be optimized are denoted by arrows.
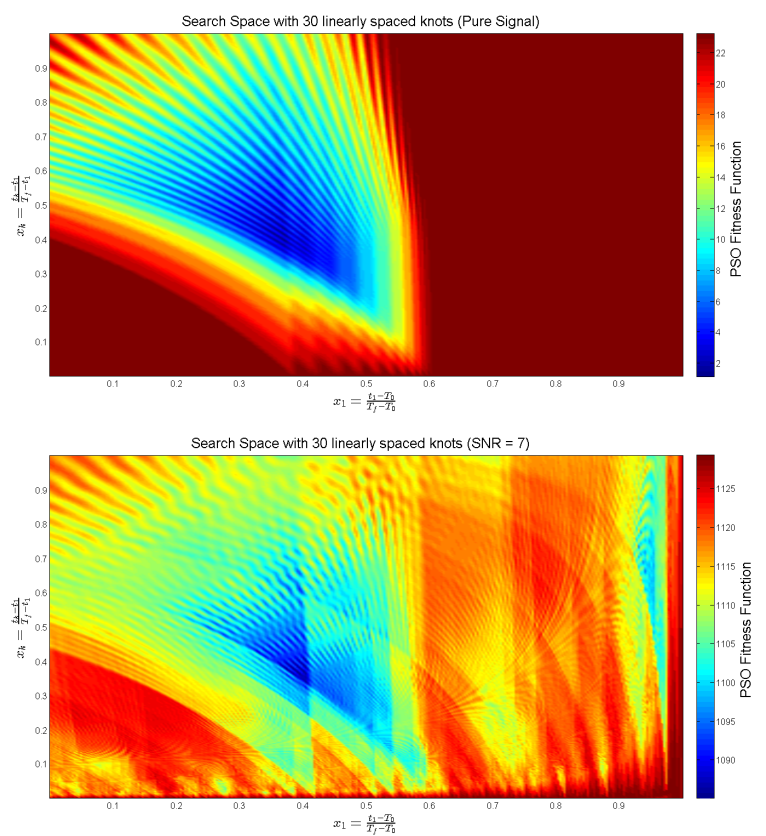

FIG. 3. Least squares fitness function (with smoothness penalty) evaluated over a $200 \times 200$ grid of the search space with a constrained knot spacing and a representative waveform, with no noise (top) and noise added (SNR $=7$, bottom).

fitness function and visualize its overall profile using just a grid search. Fig. 3 shows the fitness function for both the case of zero noise and when noise is added to the signal $(\mathrm{SNR}=7)$. (The parameters used are explained in the next subsection.) This simple case already shows the highly multi-modal nature of the fitness function. Clearly, the multi-modality can be expected to be much higher in the unconstrained knots case, invalidating the use of local numerical minimization algorithms such as gradient descent or Nelder-Mead for locating the global minimum.

\section{B. Knot Parameterization}

For both the constrained and unconstrained knot spacing schemes, there are many possible ways to parameterize a given domain partition. However, any transformation from a domain partition to a location in the search space should be invertible in order to reduce degenrate optima in the fitness function. This condition is not met, for instance, if the parameters are simply the knot locations themselves, $t_{j}, j=1,2, \ldots, k$ and each 
is varied independently by PSO. (Earlier studies [23, 24] used this scheme because of its simplicity.) There are many possible ways to impose the invertibility condition; In practice, however, certain parameterizations are more effective than others.

In our analysis we use the following invertible linear transformation (the inverse is described because it is more intuitive).

$$
\begin{aligned}
x_{1} & =\frac{t_{1}-T_{0}}{T_{f}-T_{0}} \\
x_{k} & =\frac{t_{k}-t_{1}}{T_{f}-t_{1}} \\
x_{i} & =\frac{t_{i}-t_{i-1}}{t_{i+1}-t_{i-1}}, 1<i<k
\end{aligned}
$$

The locations of the outermost (first and last) knots are encoded into the search space as distance ratios relative the endpoints of the dataset, and the remaining knots are encoded as distance ratios relative to their adjacent knots.

\section{Penalized Spline}

Preliminary tests, especially those without the constrained knot spacing, tended to overfit the noise especially at lower SNRs, as seen in the top part of Fig. 1. As a result, an overfitting penalty was introduced in the least-squares function [27], using generalized crossvalidation to fix the regulator gain $\lambda$ [24]. Given a domain partition $P$, its corresponding $\mathrm{B}$-splines $B_{j}$, and a regulator gain $\lambda$, we can analytically minimize the penalized least-squares function over all $\alpha_{i}$.

$$
R^{\prime}(\vec{\alpha})=\sum_{i=1}^{N}\left(D\left(t_{i}\right)-\sum_{j=1}^{k-3} \alpha_{j} B_{j}\left(t_{i}\right)\right)^{2}+\lambda \sum_{j=1}^{k-3} \alpha_{j}^{2}
$$

When this function is minimized, a linear system in $\vec{\alpha}$ is obtained. For a given $\lambda$ and a matrix whose columns are discrete-valued B-splines $B$, the corresponding discretely-valued best fit spline is given by

$$
\hat{s}(\lambda)=B \vec{\alpha}=B(G+\lambda I)^{-1} B^{T} \vec{D}
$$

where $G=B^{T} B$, and $\vec{D}$ and $\hat{s}(\lambda)$ are column vectors with $D_{i}=D\left(t_{i}\right)$ being the $i^{\text {th }}$ element of $\vec{D}$ and $\hat{s}_{i}(\lambda)$ being the $i^{\text {th }}$ sample of the best fit spline.

The matrix $H(\lambda)=B(G+\lambda I)^{-1} B^{T}$ which maps the data to the discretely-valued spline fit is called the influence matrix, and can be used to fix the regularization parameter $\lambda$ with numerical minimization of the generalized cross-validation[7] function,

$$
\operatorname{GCV}(\lambda)=\frac{\frac{1}{N} \sum_{i=1}^{N}\left(D_{i}-\hat{s}_{i}(\lambda)\right)^{2}}{(1-\operatorname{tr}(H) / N)^{2}}
$$

\section{PSO Topology}

The choice of neighborhood, or the topology of the swarm, used for obtaining the local best $\vec{q}_{i}$ strongly influences PSO's balance between fuller exploration of the search space and rapid convergence. (Convergence alone does not guarantee that PSO has found the global minimum or even a local one.) The most basic topology is where the neighborhood of each particle consists of every other particle. Hence all the particles are attracted to the single best location found by the swarm. This basic scheme is called the global best, or gbest, topology.

While the gbest topology converges quickly, a particular topology called the ring topology generally outperforms the gbest topology on many standard multimodal test problems, although with a significantly longer number of iterations. In the ring topology, each $q_{i}$ is determined only by comparing to two neighbors. Note that neighbors are arbitrarily assigned by index rather than by Euclidean distance - in general, a particle is not close to its neighbors in the search space, which enhances the PSO algorithm's ability to explore the search space for better local minima.

\section{E. Results}

The statistical weapon of choice for comparing the various design choices is the paired, single-tailed T-test [10]. By using a paired T-test to split the data multiple ways, the effect of any particular binary parameter can be studied while effectively holding all others constant. The results are summarized in Table II. The hypothesis that condition $A$ improves search/estimation performance compared to condition $B$ is denoted by $A>B$. The significance values of the T-test for each hypothesis, corresponding to the metrics $K$ and $J$ respectively, are denoted as $\alpha_{K}$ and $\alpha_{J}$ respectively.

Based on the fairly high $\alpha_{K}$ values, indicating a lack of statistically significant difference between the two samples being tested, it is interesting that all the design choices are found to be almost equally effective when simply locating the transient and estimating its duration. There is a slight preference, however, for constrained spacing of knots.

Unlike $K$, the metric $J$ measures the finer details of the departure between the sample values of the true signal and its estimate. It is clear, based on the extremely small $\alpha_{J}$ values, that equally-spaced knots improve the estimation capability. The data also suggest that a penalized least-squares spline fit should be used to improve estimation. There is no strong preference for either of the PSO topologies tested here, at least over the small number of iterations used in this study. We choose to use the ring topology because it is consistent with the prescription of Bratton and Kennedy while making no significant difference to computational cost, which is dominated by fitness-function evaluations (spline fitting and numerical 
minimization of $G C V(\lambda))$.

\begin{tabular}{|l|l|c|c|}
\hline$T_{\text {stop }}$ & Hypothesis & $\alpha_{K}$ (Search) & $\alpha_{J}$ (Estimation) \\
\hline 50 & Constrained spacing > Unconstrained spacing & 0.10 & $5.1 e-4$ \\
50 & Penalized fitness function > Standard fitness function & 0.62 & $8.9 e-3$ \\
50 & Ring lbest $>$ gbest & 0.36 & 0.36 \\
\hline 250 & Constrained spacing > Unconstrained spacing & 0.31 & $1.4 e-4$ \\
250 & Penalized fitness function > Standard fitness function & 0.43 & $2.7 e-3$ \\
250 & Ring lbest $>$ gbest & 0.57 & 0.49 \\
\hline
\end{tabular}

TABLE II. Significance values of each algorithm modification

\section{COHERENT NETWORK ANALYSIS}

Under a proper choice of coordinates, called the TTgauge, a planar wave perturbation in the space-time metric is defined by two linearly independent functions of time. These so-called polarization components, denoted as $h_{+}(t)$ and $h_{\times}(t)$, get linearly combined in the response of a single GW detector as a function of sky position $(\theta, \phi)$ of the plane wave source, and for compact binary coalescence signals, the orientation of the binary orbit projected on the sky $(\psi)$. The linear combination coefficients are called the antenna pattern functions of a GW detector and are usually denoted as $F_{+}(\theta, \phi, \psi)$ and $F_{\times}(\theta, \phi, \psi)$ for $h_{+}$and $h_{\times}$respectively. For a geographically distributed set of detectors, there is a time-of-flight delay associated with each detector's response which depends on $(\theta, \phi)$ alone.

\section{A. Method}

The asymptotic complexity of PSO does not explicitly depend on the dimensionality of the optimization problem. Therefore, unknown parameters such as the source location can be added without severely impacting runtime, even though each individual fitness evaluation will take longer. Two modifications to the algorithm must be made to perform coherent network analysis. Most importantly, our fitness function must be generalized to include $(\theta, \phi, \psi)$ as parameters. Also, since the azimuthal angle $\theta$ and the polarization angle $\psi$ are periodic, we abolish the standard "invisible wall" constraint on the PSO search space in favor of a torus [12]. When particles exit the search space out one wall, they reenter the search space from the opposite side. Even though this is not a standard PSO prescription, the PSO algorithm appears to be quite robust even when the global optimum is located at the edge of the search space (see Fig. 5).

In principle we can attempt to impose physical constraints on our estimates to improve signal reconstruction for special cases. For example, in the case of binary

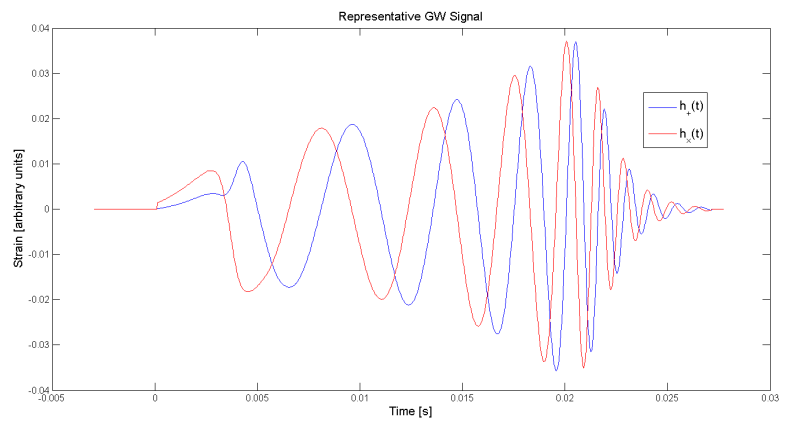

FIG. 4. GW polarization waveforms from a non-spinning binary black hole merger[5]. The actual amplitude and time scale of the signal depend on the parameters of the system such as its total mass and distance from Earth.

black hole mergers, the two linearly independent polarizations of the gravitational wave $h_{+}, h_{\times}$are related by a phase shift of a quarter period as illustrated in Fig. 4.

$$
\begin{aligned}
& h_{+}(t)=A(t) \cos (\Phi(t)) \\
& h_{\times}(t)=A(t) \sin (\Phi(t))
\end{aligned}
$$

This constraint can be modeled by cubic spline estimation of $A(t)$ and $\Phi(t)$, and has been demonstrated to work well with narrowband signals whose instantaneous frequency evolves slowly [24]. However in this study we will use only the most general of constraints: that $h_{+}$ and $h_{\times}$arrive almost at the same time and have similar durations. Two identical sets of $k$ equally-spaced knots (one for each polarization), separated by a small time difference $\Delta T \in\left(0,\left(t_{k}-t_{1}\right)\right)$ are constructed, with the time difference $\Delta T$ being optimized by PSO. Each set of knots is used for independent least-squares estimation of the $h_{+}$and $h_{\times}$polarizations. Note that when computing least-squares for a given a sky location $\theta, \phi$, the directional time delays are known and can be reversed; for simplicity we ignore the time delay in the following.

As shown in the case of a single detector, the fitness function should use a penalty term in order to improve 
performance. In the case of network analysis, we need to estimate two splines - one for each polarization - from the same set of data. Hence, there will be two penalty terms with their respective regulator gains. The automatic determination of the gains using generalized cross validation becomes a two-dimensional numerical minimization problem that severely increases computational costs. In order to avoid this computational catastrophe, we revert to the unpenalized standard least squares fitness function. With this choice, the space of solutions is still constrained by the uniformly-spaced spline knots and the agreement of more than two detectors on the two linearly-independent polarizations.

If an impingent GW signal $h_{+}, h_{\times}$is detected by the network, the data $D$ seen at $N_{d}$ detectors can be represented as in Eq. 14. Using the Moore-Penrose pseudoinverse of the second term in Eq. 14, also called the antenna pattern matrix, allows us to project the data into some two-dimensional subspace of the data [21]. A least-squares spline estimate is computed for each component; if the correct $\theta, \phi$ are selected, our spline estimates should completely contain the $h_{+}, h_{\times}$signals and agree closely with the $N_{d}$-dimensional data from the detectors. Thus our PSO fitness function to be minimized (Eq. 15) can be the least-squares comparison between the transformed $h_{+} / h_{\times}$estimates and the data at each detector, and is a function of our unknown parameters $\left(\theta, \phi, \psi, \Delta T, t_{1,+}, t_{k,+}\right)$ as desired.

$$
\begin{gathered}
D=\left[\begin{array}{cc}
h_{+} & h_{\times} \\
\vdots & \vdots
\end{array}\right]\left[\begin{array}{llll}
F_{+, 1} & F_{+, 2} & \ldots & F_{+, N_{d}} \\
F_{\times, 1} & F_{\times, 2} & \ldots & F_{\times, N_{d}}
\end{array}\right]+\text { Noise } \\
R=\sum_{\mu=1 i=1}^{\mu=N_{d} i=N}(14) \\
\left.R-\left[\begin{array}{cc}
h_{+} & h_{\times} \\
\vdots & \vdots
\end{array}\right]\left[\begin{array}{ccc}
F_{+, 1} & \ldots & F_{+, N_{d}} \\
F_{\times, 1} & \ldots & F_{\times, N_{d}}
\end{array}\right]\right)_{\mu i}^{2}
\end{gathered}
$$

\section{B. Data Pipeline}

To test the network PSO algorithm, we used a network consisting of the G1, H1, L1, and V1 interferometers, although the exact choice of detector is arbitrary. Twenty representative sky locations $(\theta, \phi, \psi=0)$ were chosen in an ad-hoc manner, and a GW signal was generated at each of four detectors using the injection engine GravEn [28] to apply appropriate directional time delays and to calculate each individual detector response. For simplicity the source inclination angle $\iota=0$ was taken to be constant and optimal.

After a signal is generated at all the detectors from a certain location, 24 independent realizations of stationary Gaussian noise are added to the data at a certain root-mean-square amplitude to give a total of 480 distinct datasets. The RMS amplitude is determined using the definition of SNR over a network in Eq. 4, where $s(t)$ is taken to be the signal with maximum norm seen by any detector in the network. We are effectively assuming that the detectors have identical sensitivities and noise floors and differ only in their position and orientation in order to assess the effect of different power distributions on the algorithm. Note that this is a simplification; G1 refers to a hypothetical $4 \mathrm{~km}$ interferometer at the location of GEO600, while H1/L1/V1 refer to the LIGO Hanford/Livingston interferometers and Virgo respectively, which have varying sensitivities in different frequency bands.

\section{Results}

In practice, we would not know if our estimates are accurate, i.e. whether PSO failed to find the global minimum of the fitness function. However we can make individual PSO runs independent by instantiating the swarm with random initial positions and velocities every time we run the algorithm on a fixed dataset. If the probability of convergence to the global minimum is $P$, then the probability of incorrectly estimating the location and waveform $(1-P)^{n}$ decreases as $n$, the number of independent PSO runs, increases. Fixing $n$ to be large enough and choosing the best of $n$ runs allows us to have some level of confidence that we have indeed estimated the true GW parameters correctly. In this study we run the PSO algorithm $n=5$ times on a dataset and choose the estimate with the optimal (minimized) fitness value. Visualizing the optimal sky location estimates over 24 noise realizations $\times 20$ sets of true GW parameters shows excellent source direction reconstruction as evidenced in Fig. 5. The true locations are denoted by crosshairs, and the twenty-four best estimates for each location are color coded for clarity.

The polarization angle $\psi$ doesn't provide additional insight into the astrophysical system; however a poorlyestimated $\psi$ can cause the resultant $h_{+}, h_{\times}$estimates to be phase shifted from the true signal. Thus, comparing the power spectra of the estimates versus the true waveforms is a better way to assess the accuracy of the spline estimates. For each of the 20 locations, we plot the maximum and minimum amplitude in each frequency bin over the 24 best estimates in Fig. 6.

In analyzing the upper/lower bound estimates on $h_{+}, h_{\times}$, we can disregard frequencies above $10^{3}-10^{4} \mathrm{~Hz}$ because of their unrealistic astrophysical interpretation and numerical noise [5]. If we concentrate on the upper bound estimates, which seem to be more coherent, we see that estimates of the principal frequency peaks at $64 \mathrm{~Hz}$ in both $h_{+}(t), h_{\times}(t)$ are poor: the corresponding peak in the estimates is consistently higher. We suspect this is a symptom of overfitting. The second frequency peak, at $\approx 197 \mathrm{~Hz}$ in $h_{+}(t)$ and $\approx 176 \mathrm{~Hz}$ in $h_{\times}(t)$, also seem at first glance to be poorly estimated. Closer inspection reveals that the peaks are consistently switched in the $h_{+}$and $h_{\times}$estimates: the general trend of $h_{+}$estimates 
has a peak at $\approx 176 \mathrm{~Hz}$ and a peak in the $h_{\times}$estimate at $\approx 197 \mathrm{~Hz}$.

We cannot explain why this seems to be consistent over multiple upper-bound estimates. Astrophysically, $h_{+}(t)$ and $h_{\times}(t)$ are coupled and the current spline fitting algorithm at present constructs them completely independently. Even with the constraints imposed by the detector network, it appears that further constraints are necessary to reduce the space of possible waveform estimates, whether through a different set of basis functions, cross-validation, or imposing physical constraints such as in the case of a binary black hole merger on top of this highly general estimation method.

\section{CONCLUSION AND FUTURE WORK}

A particle swarm optimization algorithm combined with cubic spline estimation is a promising new tool for conducting time-domain estimation of unmodeled gravitational wave transients. Regularization and constraints on the knot locations improve signal estimates in the single-detector case. Using a ring topology for the swarm adds robustness in the face of high multimodality especially in coherent network analysis, where the algorithm optimizes over all sky locations and knot positions simultaneously. Since the complexity of PSO does not depend strongly on the dimension of the optimization problem, it can accommodate additional parameters such as the $\Delta T$ parameter used to model the time offset between the set of knots used for fitting the $h_{+}$and $h_{\times}$waveforms which gives us additional flexibility in working with unmodeled transients. Future work will focus on improving the estimation in the network analysis algorithm with additional regularization, and characterization of the method as applied to actual interferometers whose noise floors are differently colored and sensitivities differ so that we may compare PSO to existing methods of transient data analysis.

\section{ACKNOWLEDGEMENTS}

This work is based upon the REU project of Calvin Leung supervised by Soumya D. Mohanty and supported by the National Science Foundation under Grant Number 1156600. Calvin wishes to thank Dr. Cristina Torres, and Dr. Matthew Benacquista, and Dr. Robert Stone for helpful discussions and making his stay at the University of Texas at Brownsville very productive.
[1] B. Aвbотt, R. Аввотt, R. Adhikari, P. Ajith, B. Allen, G. Allen, R. Amin, S. Anderson, W. AnDerson, M. ArAin, ET AL., Ligo: the laser interferometer gravitational-wave observatory, Reports on Progress in Physics, 72 (2009), p. 076901.

[2] F. Acernese, P. Amico, M. Alshourbagy, F. Antonucci, S. Aoudia, S. Avino, D. Babusci, G. BalLARdin, F. BArone, L. BArsotti, ET AL., The status of virgo, Classical and Quantum Gravity, 23 (2006), p. S635.

[3] Y. Aso, Y. Michimura, K. Somiya, M. Ando, O. Miyakawa, T. Sekiguchi, D. Tatsumi, H. Yamamoto, K. Collaboration, ET AL., Interferometer design of the kagra gravitational wave detector, Physical Review D, 88 (2013), p. 043007.

[4] J. BAKER, Astrophysical gravitational-wave sources archive.

[5] J. G. Baker, M. Campanelli, F. Pretorius, And Y. Zlochower, Comparisons of binary black hole merger waveforms, Classical and Quantum Gravity, 24 (2007), p. S25.

[6] D. Bratton And J. Kennedy, Defining a standard for particle swarm optimization, in Swarm Intelligence Symposium, 2007. SIS 2007. IEEE, IEEE, 2007, pp. 120-127.

[7] P. Craven and G. Wahba, Smoothing noisy data with spline functions, Numerische Mathematik, 31 (1978), pp. 377-403.

[8] C. De Boor, A practical guide to splines, (1978).

[9] J. Degallaix, T. Accadia, F. Acernese, M. Agathos, A. Allocca, P. Astone, G. BalLARdin, F. BArone, M. Bejger, M. Beker, Et AL., Advanced virgo status, in 9th LISA Symposium, vol. 467, 2013, p. 151.
[10] J. Devore, Probability and Statistics for Engineering and the Sciences, Cengage Learning, 2011.

[11] R. C. Eberhart And J. Kennedy, A new optimizer using particle swarm theory, in Proceedings of the sixth international symposium on micro machine and human science, vol. 1, New York, NY, 1995, pp. 39-43.

[12] A. P. ENGELBRECHT, Fundamentals of computational swarm intelligence, John Wiley \& Sons, 2006.

[13] H. W. Engl, M. Hanke, and A. Neubauer, Regularization of inverse problems, vol. 375, Springer, 1996.

[14] J. H. FRIEDMAN, Multivariate adaptive regression splines, The annals of statistics, (1991), pp. 1-67.

[15] A. GÁlvez AND A. IGlesias, Efficient particle swarm optimization approach for data fitting with free knot bsplines, Computer-Aided Design, 43 (2011), pp. 16831692.

[16] H. Grote, L. S. Collaboration, et Al., The status of geo 600, Classical and Quantum Gravity, 25 (2008), p. 114043.

[17] W. HARDLE, Applied nonparametric regression, vol. 27, Cambridge Univ Press, 1990.

[18] G. M. Harry, L. S. Collaboration, et Al., Advanced ligo: the next generation of gravitational wave detectors, Classical and Quantum Gravity, 27 (2010), p. 084006.

[19] J. HiLl, Estimating binary black hole merger signals. Brigham Young University, 2013. Senior Undergraduate Thesis.

[20] S. A. Hughes, Listening to the universe with gravitational-wave astronomy, Annals of Physics, 303 (2003), pp. 142-178.

[21] S. Klimenko, S. Mohanty, M. Rakhmanov, And G. Mitselmakher, Constraint likelihood analysis for a 

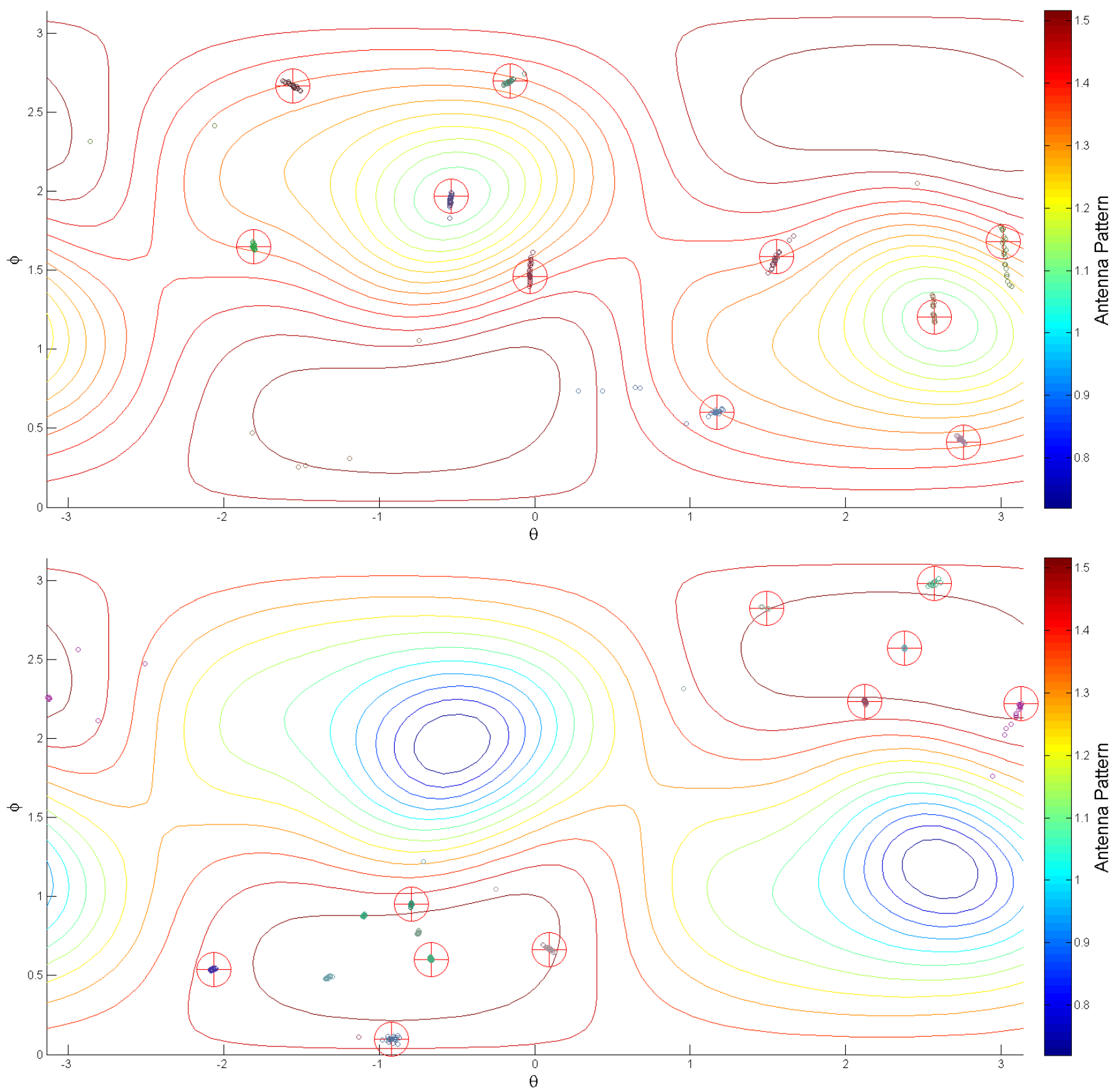

FIG. 5. Note that the sizes of the crosshairs are arbitrary and that the Mercator projection increases distances towards the top and bottom edges of the skymap. Locations 1-10/11-20 are visualized on the top and bottom skymaps respectively.

network of gravitational wave detectors, Physical Review D, $72(2005)$.

[22] S. D. Mohanty, First Transient Optical Robotic Observatory of the South (TOROS) Workshop.

[23] — Particle swarm optimization and regression analysis II, The Astronomical Review, 7 (2012), pp. 4-25.

$[24] \longrightarrow$, Detection and estimation of unmodeled narrowband nonstationary signals: application of particle swarm optimization in gravitational wave data analysis, in Proceedings of the 2014 conference companion on Genetic and evolutionary computation companion, ACM, 2014, pp. $173-174$.
[25] C. D. OтT, The gravitational-wave signature of corecollapse supernovae, Classical and Quantum Gravity, 26 (2009), p. 063001.

[26] Y. Pan, A. Buonanno, A. Taracchini, L. E. KidDer, A. H. Mroué, H. P. Pfeiffer, M. A. Scheel, AND B. SzILÁGYI, Inspiral-merger-ringdown waveforms of spinning, precessing black-hole binaries in the effectiveone-body formalism, Physical Review D, 89 (2014), p. 084006.

[27] D. Ruppert, M. P. Wand, and R. J. Carroll, Semiparametric Regression, Cambridge Series in statistical and probabilistic mathematics, 2003. 


\begin{tabular}{|c|c|c|c|c|c|c|}
\hline$\#$ & $\theta$ & $\phi$ & G1 & $H 1$ & $L 1$ & $V 1$ \\
\hline 1 & -1.5550 & 2.6660 & 15.0000 & 9.4052 & 8.0356 & 13.5700 \\
\hline 2 & -0.1587 & 2.6970 & 9.5997 & 15.0000 & 10.7890 & 10.1129 \\
\hline 3 & -0.0317 & 1.4600 & 13.0234 & 9.5016 & 8.5941 & 15.0000 \\
\hline 4 & -1.8090 & 1.6500 & 5.9002 & 12.4994 & 15.0000 & 10.5356 \\
\hline 5 & -0.5395 & 1.9670 & 10.0854 & 10.0589 & 8.4629 & 15.0000 \\
\hline 6 & 1.5550 & 1.5870 & 3.9634 & 11.8893 & 15.0000 & 8.3281 \\
\hline 7 & 3.0150 & 1.6820 & 12.8837 & 8.7547 & 8.5958 & 15.0000 \\
\hline 8 & 2.7610 & 0.4125 & 10.5371 & 15.0000 & 11.1817 & 11.4506 \\
\hline 9 & 1.1740 & 0.6029 & 15.0000 & 5.2102 & 4.4639 & 14.2310 \\
\hline 10 & 2.5700 & 1.2060 & 11.7637 & 9.2022 & 9.8249 & 15.0000 \\
\hline 11 & 1.4910 & 2.8240 & 12.8450 & 15.0000 & 13.1823 & 10.1846 \\
\hline 12 & 2.5700 & 2.9830 & 15.0000 & 12.7113 & 10.6612 & 13.3431 \\
\hline 13 & 3.1300 & 2.2210 & 14.7354 & 7.6400 & 7.6828 & 15.0000 \\
\hline 14 & 2.1260 & 2.2330 & 11.4353 & 12.2855 & 15.0000 & 11.9645 \\
\hline 15 & 2.3800 & 2.5700 & 15.0000 & 13.0200 & 12.9602 & 14.1929 \\
\hline 16 & -0.7933 & 0.9520 & 14.2127 & 11.7801 & 15.0000 & 14.3014 \\
\hline 17 & 0.0952 & 0.6660 & 15.0000 & 6.9223 & 6.0027 & 14.6210 \\
\hline 18 & -0.9203 & 0.0952 & 15.0000 & 13.8360 & 11.3846 & 13.7121 \\
\hline 19 & -2.0630 & 0.5395 & 9.3972 & 15.0000 & 12.9595 & 5.4050 \\
\hline 20 & -0.6660 & 0.6029 & 15.0000 & 12.1867 & 12.2182 & 14.4190 \\
\hline
\end{tabular}

TABLE III. The expected SNR at each detector as shown above for our twenty injection locations, with $\max (S N R)=15$ and $\phi, \iota=0$.

[28] A. L. Stuver AND L. S. Finn, Graven: software for the simulation of gravitational wave detector network response, Classical and Quantum Gravity, 23 (2006), p. S799.

[29] S. Weinberg AND R. Dicke, Gravitation and cosmology: principles and applications of the general theory of relativity, American Journal of Physics, 41 (1973), pp. 598-599.

[30] D. H. Wolpert and W. G. Macready, No free lunch theorems for optimization, Evolutionary Computation, IEEE Transactions on, 1 (1997), pp. 67-82. 

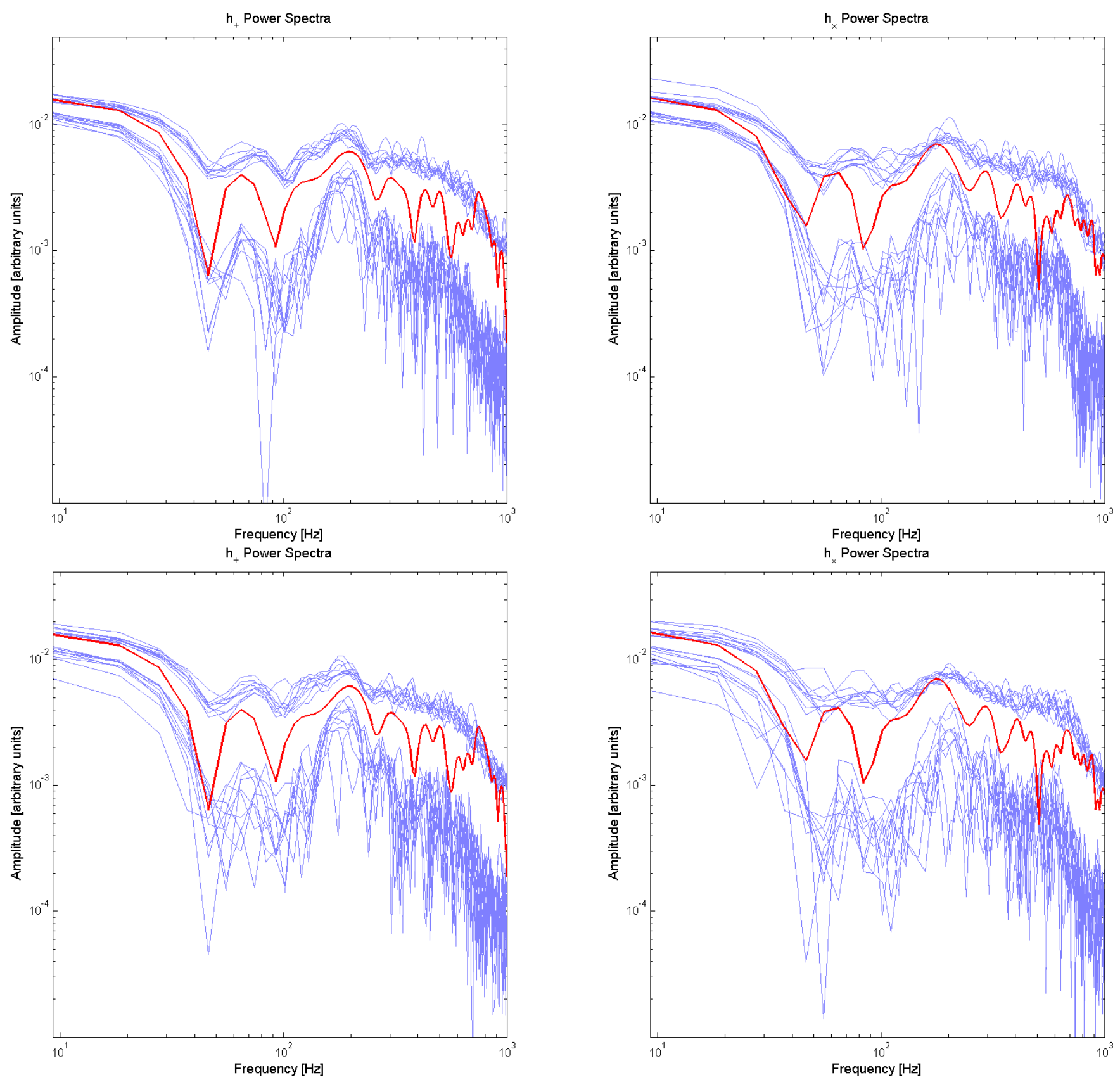

FIG. 6. Power spectral densities of the estimated waveforms compared with the true ones (red curve). The minimum and maximum value obtained across 24 noise realizations is plotted at each frequency. Different colors correspond to different sky locations of the true source (1-10/11-20 are visualized on the top and bottom respectively). 